\title{
Decomposition method using He's polynomials for Kdv Boussinesq and coupled Kdv Boussinesq equations
}

\author{
Jamshad Ahmad ${ }^{1}$ and Mariyam Mushtaq ${ }^{2}$ \\ ${ }^{1}$ Department of Mathematics, Faculty of Sciences, University of Gujrat, Pakistan \\ ${ }^{2}$ Department of Mathematics, GC Women University Sialkot, Pakistan
}

Received: 11 April 2016, Accepted: 26 February 2017

Published online: 20 October 2017.

\begin{abstract}
This paper obtains the solitary wave solutions of two different forms of Boussinesq equations that model the study of shallow water waves in lakes and ocean beaches. The decomposition method using He's polynomials is applied to solve the governing equations. The travelling wave hypothesis is also utilized to solve the generalized case of coupled Boussinesq equations, and, thus, an exact soliton solution is obtained. The results are also supported by numerical simulations.
\end{abstract}

Keywords: Decomposition method, He's polynomials, cubic Boussinesq equation, coupled Boussinesq equations.

\section{Introduction}

In recent years, remarkable developments have taken place in the study of nonlinear evolutionary partial differential equations. It is realized that many such equations possess special solutions in the form of pulses which retain their shapes and velocities after interacting with each other. Such solutions are called solitons. Many equations admitting soliton solutions are as follows: sine Gordon and double sine Gordon equations, Schrodinger equation, and KDV, MKDV, and complex modified KDV equations many research works have been done on these equations. Most of the current research is directed to solve coupled nonlinear systems analytically and numerically. Solitons are of great interest in many physical areas, as, for example, in dislocation theory of crystals, plasma and fluid dynamics, magneto hydrodynamics, laser and fiber optics, and the study of the water waves. Many research works on Boussinesq equation have been developed analytical solution of this equation was studied by many authors [1-32], such as the construction of soliton solutions using the bilinear form [4], multiple soliton solutions for the GB equation using a simplified version of Hirota method [27] and decomposition method [28]. Construction of soliton solutions and periodic solution of Boussinesq equation by modified decomposition method are given in [29, 30]. A variational iteration method is developed for GB equation [31]. A solitary wave solution of the Boussinesq equation with power law nonlinearity is derived in [32]. Many numerical methods have been developed for solving the Boussinesq equation, suchas Petrov-Galerkin method [19]. Mohebbi and Asgari [26] also have solved the GB equation using a fourth order time stepping schemes with combination of discrete Fourier transform. Split step Fourier method is also used to solve Boussinesq-type equations.

\section{Decomposition method using He's polynomials}

To illustrate the basic concept of modified decomposition method, we consider the following general differential equation

$$
L(u)+N(u)=g(x)
$$


where $\mathrm{L}$ is the liner operator and $\mathrm{N}$ is the nonlinear operator and $\mathrm{g}(\mathrm{x})$ is the homogeneous term. According to the $\mathrm{ADM}$ we construct the

$$
u_{n+1}=u_{n}-L_{t}^{-1}\{N(u)+g(x)\},
$$

where $L_{t}^{-1}=\int_{0}^{t} d t$, . The embedding parameter $p \in(0,1]$ can be considered as an Expanding parameters. The homotopy perturbation method uses the homotopy parameter $p$ as an expanding parameter to obtain

$$
u=\sum_{n=0}^{\infty} p^{n} u_{n}=u_{0}+p u_{1}+p^{2} u_{2}+p^{3} u_{3}+\cdots
$$

If $p \rightarrow 1$, the approximate solution of the form,

$$
f=\lim _{p \rightarrow 1} u=\sum_{n=0}^{\infty} u_{n}
$$

It is well known that series (4) is convergent for most of the cases and also the rate of convergence is dependent on L (u). We assume that (4) has a unique solution. The comparisons of like powers of $p$ give solutions of various orders. In sum, according to, He's considers the solution $u(x)$, of the homotopy equation in a series of $p$ as follows.

$$
u(x)=\sum_{n=0}^{\infty} p^{n} u_{n}=u_{0}+p u_{1}+p^{2} u_{2}+p^{3} u_{3}+\cdots
$$

and the method consider the nonlinear term $N(u)$, as

$$
N(u)=\sum_{n=0}^{\infty} p^{n} H_{n}=H_{0}+p H_{1}+p^{2} H_{2}+p^{3} H_{3}+\cdots,
$$

where $H_{n}$ are so called He's polynomials which can be calculated by using the formula

$$
H_{n}\left(u_{0}, u_{1}, u_{2}, \ldots\right)=\frac{1}{n !} \frac{\partial^{n}}{\partial p^{n}}\left[N\left(\sum_{i=0}^{\infty} p^{i} u_{i}\right)\right]_{p=0}, n=0,1,2,3, \ldots
$$

The successive approximation $u_{n+1}, n \geq 0$ of the solution of $\mathrm{u}$ will be obtained by selective function $u_{0}$. Consequently the solution is given by $u=\lim _{n \rightarrow \infty} u_{n}$.

\section{Numerical applications}

In this section, we apply the decomposition method using He's polynomials for solving Boussinesq and coupled Boussinesq equations.

Example 1. Consider the cubic KDV Boussinesq equation

$$
u_{t t}-u_{x x}+2\left(u^{3}\right)_{x x}-u_{x x x x}=0
$$

with subject to initial condition

$$
u(x, 0)=\frac{1}{x}, u_{t}(x, 0)=-\frac{1}{x^{2}}
$$


According to the above procedure

$$
\begin{aligned}
& u(x, t)=\frac{1}{x}-\frac{1}{x^{2}} t+p L_{t}^{-1} L_{t}^{-1}\left\{\sum_{n=0}^{\infty} p^{n}\left(u_{n x x}\right)-2 \sum_{n=0}^{\infty} p^{n}\left(u^{3}\right)_{n x x}+\sum_{n=0}^{\infty} p^{n}\left(u_{n x x x x}\right)\right\} \\
& u(x, t)=\frac{1}{x}-\frac{1}{x^{2}} t+p L_{t}{ }^{-1} L_{t}-1\left\{\sum_{n=0}^{\infty} p^{n}\left(u_{n x x}\right)-2 \sum_{n=0}^{\infty} p^{n}\left(H_{n}(u)\right)+\sum_{n=0}^{\infty} p^{n}\left(u_{n x x x x}\right)\right\}
\end{aligned}
$$

Equating the like power components of $p$, we get

$$
\begin{aligned}
p^{0}: u_{0} & =\frac{1}{x}-\frac{1}{x^{2}} t \\
p^{1}: u_{1} & =L_{t}^{-1} L_{t}^{-1}\left\{u_{0 x x}-2 H_{0}+u_{0 x x x x}\right\} \\
& =\frac{t^{2}}{x^{3}}-\frac{t^{3}}{x^{4}}-\frac{180 t^{4}}{12 x^{7}}+\frac{84 t^{5}}{20 x^{8}}=\frac{t^{2}}{x^{3}}-\frac{t^{3}}{x^{4}}+\text { small terms }, \\
p^{2}: u_{2} & =L_{t}^{-1} L_{t}^{-1}\left\{u_{1 x x}-2 H_{1}+u_{1 x x x x}\right\} \\
& =\frac{t^{4}}{x^{5}}-\frac{t^{6}}{x^{7}}+\text { small terms }
\end{aligned}
$$

and so on, summing all components of $\mathrm{u}$, we get

$$
\begin{aligned}
u(x, t) & =u_{0}+u_{1}+u_{2}+\cdots, \\
& =\frac{1}{x}-\frac{1}{x^{2}} t+\frac{t^{2}}{x^{3}}-\frac{t^{3}}{x^{4}}+\frac{t^{4}}{x^{5}}-\frac{t^{6}}{x^{7}}+\text { small terms }, \\
& =\frac{1}{x+t} .
\end{aligned}
$$

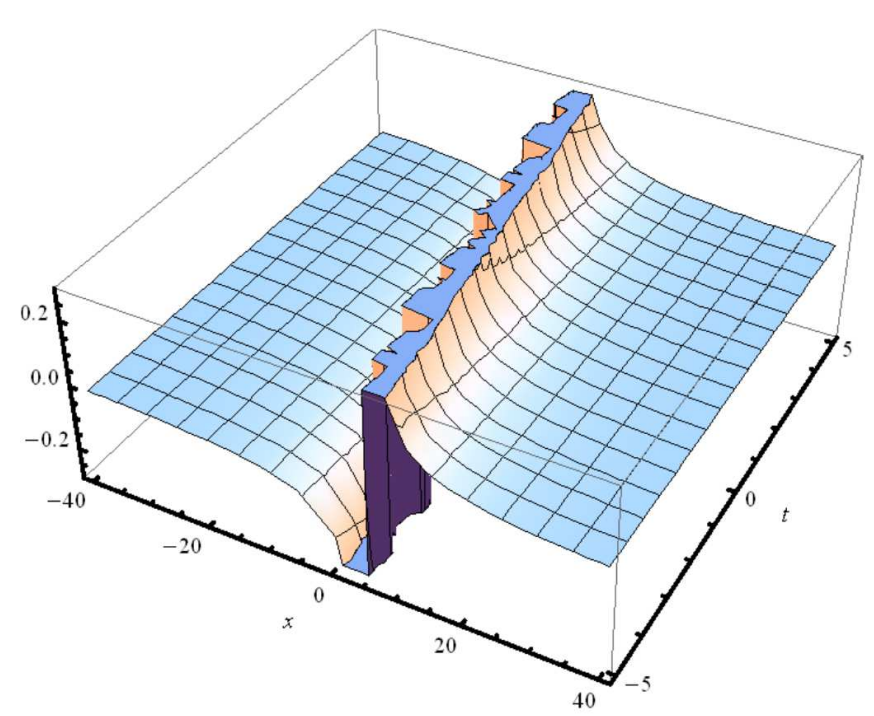

Fig. 1: behavior of $u(x, t)$ with $-40 \leq x \leq 40$ and $-5 \leq t \leq 5$. 
Example 2. Consider the coupled Boussinesq equations

$$
\begin{gathered}
u_{t}+v_{x}+u u_{x}=0, \\
v_{t}+(v u)_{x}+u_{x x x}=0,
\end{gathered}
$$

with subject to initial conditions

$$
\begin{gathered}
u(x, 0)=\frac{\lambda}{k}+2 k \tanh (k x), \\
v(x, 0)=2 k^{2} \operatorname{sech}^{2}(k x) .
\end{gathered}
$$

According to the above procedure

$$
\begin{aligned}
& u(x, t)=\frac{\lambda}{k}+2 k \tanh (k x)-p L_{t}^{-1}\left\{\sum_{n=0}^{\infty} p^{n}\left(v_{n x}\right)+\sum_{n=0}^{\infty} p^{n}\left(u_{n} u_{n x}\right)\right\}, \\
& v(x, t)=2 k^{2} \operatorname{sech}^{2}(k x)-p L_{t}^{-1}\left\{\sum_{n=0}^{\infty} p^{n}(v u)_{n x}-\sum_{n=0}^{\infty} p^{n}\left(u_{n x x x}\right)\right\}, \\
& u(x, t)=\frac{\lambda}{k}+2 k \tanh (k x)-p L_{t}^{-1}\left\{\sum_{n=0}^{\infty} p^{n}\left(v_{n x}\right)+\sum_{n=0}^{\infty} p^{n}\left(H_{n}\right)\right\}, \\
& v(x, t)=2 k^{2} \operatorname{sech}^{2}(k x)-p L_{t}^{-1}\left\{\sum_{n=0}^{\infty} p^{n}\left(M_{n}\right)-\sum_{n=0}^{\infty} p^{n}\left(u_{n x x x}\right)\right\},
\end{aligned}
$$

where $H_{n}$ and $M_{n}$ are nonlinear terms, equating like power components of $\mathrm{p}$, we get

$$
\begin{aligned}
p^{0}: u_{0} & =\frac{\lambda}{k}+2 k \tanh (k x), \\
p^{0}: v_{0} & =2 k^{2} \operatorname{sech}^{2}(k x), \\
p^{1}: u_{1} & =-L_{t}{ }^{-1}\left\{v_{0 x}+H_{0}\right\} \\
& =4 k^{3} \operatorname{sech}^{2}(k x) \tanh (k x) t-2 \lambda k \operatorname{sech}^{2}(k x) t-4 k^{3} \operatorname{sech}^{2}(k x) \tanh (k x) t, \\
& =-2 \lambda k \operatorname{sech}^{2}(k x) t, \\
p^{1}: v_{1} & =-L_{t}^{-1}\left\{M_{0}+u_{0 x x x}\right\} \\
& =-4 k^{4} \operatorname{sech}^{4}(k x) t+4 \lambda k^{2} \operatorname{sech}^{2}(k x) \tanh (k x) t+8 k^{4} \operatorname{sech}^{2}(k x) t-8 k^{4} \operatorname{sech}^{4}(k x) t-8 k^{4} \operatorname{sech}^{2}(k x) t+12 k^{4} \operatorname{sech}^{4}(k x) t, \\
& =4 \lambda k^{2} \operatorname{sech}^{2}(k x) \tanh (k x) t .
\end{aligned}
$$

and so on, summing all components of $u$ and $v$, we get

$$
\begin{aligned}
& u(x, t)=\frac{\lambda}{k}+2 k \tanh (k x)-2 \lambda \operatorname{sech}^{2}(k x) t-\ldots, \\
& u(x, t)=\frac{\lambda}{k}+2 k \tanh (k x-\lambda t) . \\
& v(x, t)=2 k^{2} \operatorname{sech}^{2}(k x)+4 \lambda k^{2} \operatorname{sech}^{2}(k x) \tanh (k x) t+\ldots, \\
& v(x, t)=2 k^{2} \operatorname{sech}^{2}(k x-\lambda t) .
\end{aligned}
$$




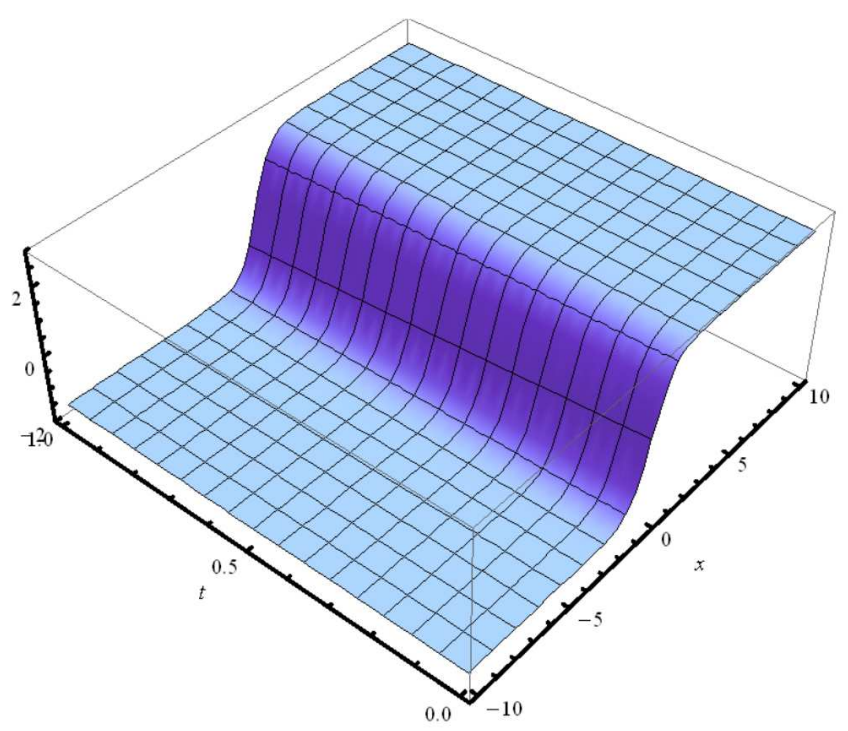

Fig. 2: Behavior of $\mathrm{u}(\mathrm{x}, \mathrm{t})$ with $-10 \leq x \leq 10$ and $0 \leq t \leq 1$ and $k=1, \lambda=0.5$.

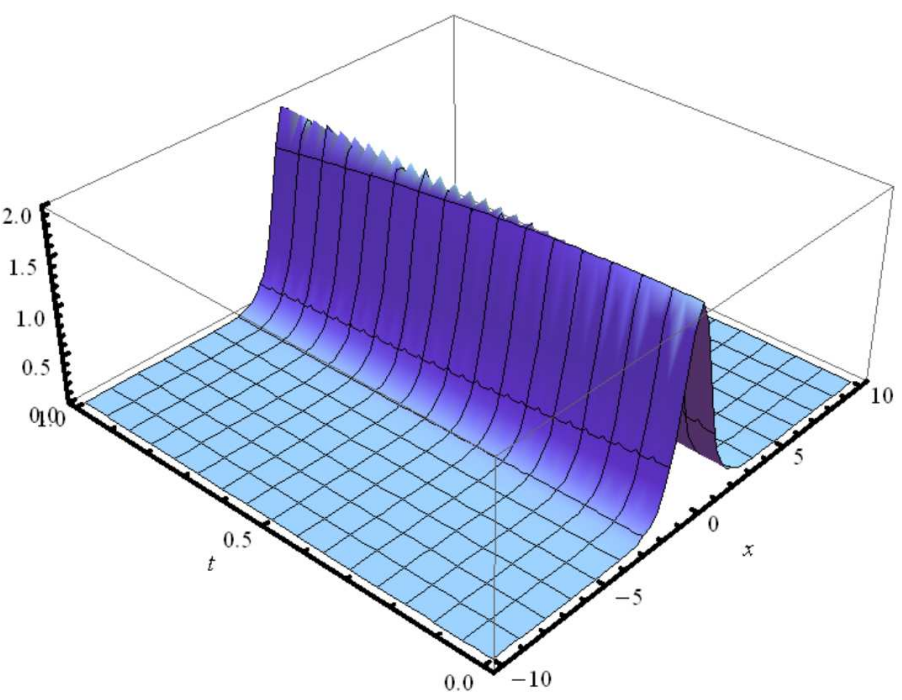

Fig. 3: Behavior of $\mathrm{v}(\mathrm{x}, \mathrm{t})$ with $-10 \leq x \leq 10$ and $0 \leq t \leq 1$ and $k=1, \lambda=0.5$.

\section{Conclusion}

There are two main goals that we aimed for this work. The first is to show the power of the modified decomposition method using He's polynomials and its significant features. The second is to employ this method to obtain rational solutions of nonlinear dispersive equations. It is obvious that the method gives rapid convergent successive approximations without any restrictive assumptions or transformation that may change the physical behavior of the problem. Moreover, the decomposition method reduces the size of calculations by not requiring the tedious working. The 
cubic Boussinesq and coupled Boussinesq equations were examined for rational solutions only and soliton solution are obtained. The desired solutions were obtained rapidly and in a direct way.

\section{Competing interests}

The authors declare that they have no competing interests.

\section{Authors' contributions}

All authors have contributed to all parts of the article. All authors read and approved the final manuscript.

\section{References}

[1] Wazwaz, A. The variational iteration method for rational solutions for $\mathrm{KdV}, \mathrm{K}(2 ; 2)$, Burgers, and cubic Boussinesq equations, Journal of Computational and Applied Mathematics, 207(1), pp. 18-23 (2007).

[2] J. Ahmad, M. Mushtaq and N.Sajjad, Exact Solution Of Whitham-Broer-Kaup Shallow Water Wave Equations, Journal of Science and Arts, 1(30), pp. 5-12, 2015.

[3] Girgis, L., Zerrad, E. and Biswas, A. Solitary wave solutions of the Peregrine equation, International Journal of Oceans and Oceanography 4(1), pp. 45-54 (2010).

[4] G. Domairry, A. G. Davodi, and A. G. Davodi,vSolutions for the double sine-Gordon equation by Exp-function, tanh, and extended tanh methods, Numerical Methods for Partial Differential Equations, vol. 26, no. 2, pp. 384-398, 2010.

[5] Jawad, A.J.M., Petkovic, M.D. and Biswas, A. Soliton solutions of Burgers equations and perturbed Burgers equation, Applied Mathematics and Computation, 216(11), pp. 3370-3377 (2010).

[6] Jawad, A.J.M., Petkovic, M.D. and Biswas, A. Soliton solutions of a few nonlinear wave equations, Applied Mathematics and Computation, 216(9), pp. 2649-2658 (2010).

[7] Li, B., Chen, Y. and Zhang, H.Q. Explicit exact solutions for some nonlinear partial differential equations with nonlinear terms of any order, Czechoslovak Journal of Physics, 53(4), pp. 283-295 (2003)

[8] Malfliet, W. Solitary wave solutions of nonlinear wave equations, American Journal of Physics, 60, pp. 650-654 (1992).

[9] Mousa, M.M. and Kaltayev, A. Constructing approximate and exact solutions for Boussinesq equations using homotopy perturbation Pade technique, World Academy of Science, Engineering and Technology, 38, pp. 3730-3740 (2009).

[10] Ugurlu, Y. and Kaya, D. Exact and numerical solutions of generalized Drinfeld-Sokolov equations, Physics Letters A, 372(16), pp. 2867-2873 (2008).

[11] Wu, L., Chen, S. and Pang, C. Traveling wave solutions for generalized Drinfeld-Sokolov equations, Applied Mathematical Modeling, 33(11), pp. 4126-4130 (2009).

[12] Ganji, D.D., Nourollahi, M. and Rostamian, M. A comparison of variational iteration method with Adomian's decomposition method in some highly nonlinear equations, International Journal of Science and Technology, 2(2), pp. 179-188 (2007)

[13] Wolfram. The Mathematica, fifth ed., Wolfram Media/Cambridge University Press, Champaign, IL 61820, USA Book (2003).

[14] He, J.H. Comparison of homotopy perturbation method and homotopy analysis method, Applied Mathematics and Computation, 156, pp. 527-539 (2004).

[15] Malfliet, W. The tanh method: a tool for solving certain classes of nonlinear evolution and wave equations, Journal of Computational and Applied Mathematics, 164-165, pp. 529-541 (2004).

[16] Wazwaz, A., Partial Differential Equations and Solitary Waves Theory, Springer Verlag, New York, NY, USA (2009).

[17] Wazwaz, A. The Cole-Hopf transformation and multiple soliton solutions for the integrable sixth-order Drinfeld-SokolovSatsuma-Hirota equation, Applied Mathematics and Computation, 207(1), pp. 248-255 (2009).

[18] Kara, A.H. and Mahomed, F.M. 'Noether-type symmetries and conservation laws via partial Lagrangians', Nonlinear Dynamics, 45(3-4), pp. 367-383 (2006). 
[19] V. S. Manoranjan, A. R. Mitchell, and J. Ll. Morris, Numerical solutions of the good Boussinesq equation, Society for Industrial and Applied Mathematics, vol. 5, no. 4, pp. 946-957, 1984.

[20] S. S. Ray, Soliton solutions for time fractional coupled modified KdV equations using new coupled fractional reduced differential transform method, Journal of Mathematical Chemistry, vol. 51, no. 8, pp. 2214-2229, 2013.

[21] S. Zhou and X. Cheng, Numerical solution to couple nonlinear Schrodinger equations on unbounded domains, Mathematics and Computers in Simulation, vol. 80, no. 12, pp. 2362-2373, 2010.

[22] H. Hu, L. Liu, and L. Zhang, New analytical position, negaton and complexiton solutions of a coupled KdV-mKdV system, Applied Mathematics and Computation, vol. 219, no. 11,pp. 5743- 5749, 2013.

[23] A. G. Bratsos, A second order numerical scheme for the solution of the one-dimensional Boussinesq equation, Numerical Algorithms, vol. 46, no. 1, pp. 45-58, 2007.

[24] A. G. Bratsos, Ch. Tsitouras, and D. G. Natsis, Linearized numerical schemes for the Boussinesq equation, Applied Numerical Analysis and Computational Mathematics, vol. 2, no. 1, pp. 34-53, 2005.

[25] M. S. Ismail and A. G. Bratsos, A predictor-corrector scheme for the numerical solution of the Boussinesq equation, Journal of Applied Mathematics \& Computing, vol. 13, no. 1-2, pp. 11-27, 2003.

[26] A. Mohebbi and Z. Asgari, Efficient numerical algorithms for the solution of good Boussinesq equation in water wave propagation, Computer Physics Communications, vol. 182, no. 12, pp. 2464-2470, 2011.

[27] A.-M. Wazwaz, Multiple-soliton solutions for the Boussinesq equation, Applied Mathematics and Computation, vol. 192, no. 2, pp. 479-486, 2007.

[28] B. S. Attili, The Adomian decomposition method for solving the Boussinesq equation arising in water wave propagation, Numerical Methods for Partial Differential Equations, vol. 22, no. 6, pp. 1337-1347, 2006.

[29] A.-M. Wazwaz, New travelling wave solutions to the Boussinesq and the Klein-Gordon equations, Communications in Nonlinear Science and Numerical Simulation, vol. 13, no. 5, pp. 889-901, 2008.

[30] A. M.Wazwaz, Construction of soliton solutions and periodic solutions of the Boussinesq equation by the modified decomposition method, Chaos, Solitons and Fractals, vol. 12, no. 8, pp. 1549-1556, 2001.

[31] A. Yildirim and S. T. Mohyud-Din, A variational approach for soliton solutions of good Boussinesq equation, Journal of King Saud University, vol. 22, no. 4, pp. 205-208, 2010.

[32] A. Biswas, D. Milovic, and A. Ranasinghe, Solitary waves of Boussinesq equation in a power law media, Communications in Nonlinear Science and Numerical Simulation, vol. 14, no. 11,pp. 3738-3742, 2009. 